\title{
El «NO» de Ricardo Lagos a la invasión de Irak en 2003: el proceso de toma de decisiones de politica exterior en Chile
}

\author{
Ricardo Lagos says «NO» to Irak invasion in 2003: \\ the foreign policy decision-making process in Chile \\ Cristóbal Bywaters C.*
}

\section{Resumen}

El artículo analiza el proceso de toma de decisiones de política exterior que llevó a que Chile no apoyara la invasión estadounidense de Irak en 2003. La decisión fue tomada por el Presidente Ricardo Lagos más de un mes antes de ser comunicada a Estados Unidos y se basó en sus percepciones de que la guerra era inevitable y de que las pruebas de la existencia de armas de destrucción masiva eran inconsistentes. Estas percepciones se confirmaron mediante un razonamiento analógico comparando las exposiciones de Colin Powell y Adlai Stevenson en el Consejo de Seguridad de las Naciones Unidas. La investigación se nutre de entrevistas y testimonios de algunos de los principales actores involucrados en el proceso decisorio.

PALABRAS ClAVE: invasión de Irak, percepciones sobre la guerra, toma de decisiones de política exterior, política exterior chilena, Ricardo Lagos.

\section{Abstract}

This article analyzes the foreign policy decision-making process that led Chile not to support the 2003 invasion of Iraq. It states that Pre-

Investigador, Facultad de Ciencias Sociales de la Universidad Alberto Hurtado. <bywaters. cristobal@gmail.com>

Recibido el 21 de octubre de 2013; aceptado el 10 de diciembre de 2013 
sident Ricardo Lagos made the decision a month before notifying the United States. The decision was based on Lagos' perceptions about the inevitability of the war and the inconsistence of evidence regarding the existence of weapons of mass destruction in Iraq. These perceptions were later confirmed comparing via analogical reasoning Colin Powell's and Adlai Stevenson's presentations before the UN Security Council. This research is based on interviews and statements made by some of the main actors involved in the decision-making process.

KEYWORDS: Invasion of Iraq, foreign policy decision-making, perceptions regarding the inevitability of war, Chilean foreign policy, Ricardo Lagos. 
Cristóbal Bywaters • El «NO» de Ricardo Lagos a la invasión a Irak en 2003...

\section{INTRODUCCIÓN}

En 1990, luego de 17 años de autoritarismo y relativo aislamiento internacional $^{1}$, Chile inició su re-inserción política internacional, al mismo tiempo que recuperaba su régimen democrático y la Guerra Fría llegaba a su fin. A partir de entonces se inició un período de excepcionales relaciones con Estados Unidos como resultado de la adhesión pragmática de Chile a los pilares del «nuevo orden mundial» (Fermandois, 2005). El eje de la relación bilateral pasó a ser la negociación de un tratado de libre comercio cuyas rondas se dilataron durante más de una década debido a diversos factores (Porras, 2003). Pese a estos obstáculos, la negociación culminó con éxito en diciembre de 2002, pocos días antes de que Chile ingresara al Consejo de Seguridad de las Naciones Unidas por cuarta vez en su historia.

Por aquellos días, la agenda del Consejo se centraba en la pretensión del gobierno del Presidente George W. Bush (2001-2009) de invadir Irak bajo el supuesto de que disponía de armas de destrucción masiva y de que según informes de inteligencia estadounidenses, Saddam Hussein prestaba apoyo a grupos terroristas internacionales. Por distintos canales, la diplomacia Washington hizo saber al gobierno del Presidente Ricardo Lagos (2000-2006) que una postura desfavorable a la invasión pondría en riesgo el camino que aún

Ver Muñoz (1986) y Ross (2005). le faltaba por recorrer a la aprobación del tratado de libre comercio. De este modo, entraban en conflicto dos de los principales objetivos de la política exterior del gobierno de Lagos: la suscripción del tratado de libre comercio y la adopción de un alto perfil en la política internacional ${ }^{2}$.

Contraviniendo las expectativas que podría generar un país que tradicionalmente se ha auto-descrito como pequeño y distante, Chile jugó un rol activo en el polarizado debate entre las potencias, buscando contribuir a la consecución de consensos que permitieran salvaguardar la arquitectura multilateral de las Naciones Unidas. Junto a México -el otro país latinoamericano representado en el Consejo-, Chile adoptó una actitud equidistante respecto de las posiciones polares de Estados Unidos y Francia, respectivamente. Si bien por separado ambas posiciones constituían argumentos moderados, en conjunto representaban una férrea oposición a la actitud unilateralista de Estados Unidos y un acérrimo apoyo al multilateralismo de las Naciones Unidas (Bywaters, 2013).

En una conversación telefónica del día 12 de marzo de 2003, ante la pregunta de Bush de si Chile formaría parte de la coalición de los dispuestos ${ }^{3}$

2 Esto también se expresó en la presencia chilena en la MINUSTAH y la candidatura y elección de José Miguel Insulza como Secretario General de la Organización de los Estados Americanos.

3 Estados Unidos, Gran Bretaña, España y Bulgaria, entre otros. 
liderada por Estados Unidos a pesar de no apoyar la segunda resolución que Washington planeaba presentar como último intento, Lagos respondió: "Señor Presidente, Chile y Estados Unidos tienen una estrecha relación. Pero si usted forma una coalición fuera del Consejo de Seguridad, Chile no puede participar» ${ }^{4}$ (Lagos, 2012: 224). Al no tener asegurados los votos necesarios para ser aprobada y ante el esperable veto francés, la segunda resolución nunca fue sometida a votación. Una semana después de aquella conversación, Estados Unidos y los dispuestos desplegaron su fuerza contra Bagdad sin la autorización de Naciones Unidas.

Existen varias interpretaciones sobre las razones por las cuales Chile no apoyó la invasión ${ }^{5}$. La versión oficial afirma que la posición chilena se fundamentó en los principios de su política exterior (Muñoz, 2004; Walker, 2006). Una interpretación crítica es la de Hernán Felipe Errázuriz (2003), quien afirma que Lagos privilegió una política de prestigio por sobre el interés nacional de apoyar a Estados Unidos. Existe, además, una interpretación no escrita pero bien difundida entre los círculos políticos y diplomáticos (Núñez, 2012) según la cual Lagos no habría permitido que alguien le dijera qué hacer -ni

$4 \quad$ Todas las citas de textos originalmente en inglés han sido traducidas por el autor.

5 La edición número 142 del año 2003 de Estudios Internacionales fue dedicado exclusivamente al análisis de esta coyuntura. siquiera el Presidente de los Estados Unidos.

Aunque intuitiva, esta última interpretación acierta al sugerir que la decisión fue tomada por Ricardo Lagos. En efecto, durante su administración se evidenció una alta concentración de poder en la toma de decisiones de política exterior en la figura del Presidente, que se vio exacerbada por las características particulares de la personalidad de Lagos y el contexto nacional e internacional que predominó durante la discusión sobre la cuestión de Irak. Todos los entrevistados ${ }^{6}$ en el transcurso de esta investigación coincidieron en que fue Lagos y no otro quien tomó la decisión. Lo anterior permite, desde nuestra perspectiva, explicar las razones que llevaron a que Chile no apoyara la

6 Entre mayo de 2012 y julio de 2013 se realizaron 13 entrevistas semi-estructuradas a los siguientes actores involucrados en el proceso de toma de decisiones: Ricardo Lagos (Presidente de la República); Juan Gabriel Valdés (Embajador ante las Naciones Unidas); Ernesto Ottone, Guillermo Campero, Carlos Vergara, Enrique Paris y Fernando Reyes Matta (asesores presidenciales); Carlos Portales (Director General de Política Exterior); Jaime Gazmuri, Ricardo Núñez, Carlos Ominami y José Antonio Viera Gallo (senadores socialistas); y Edgardo Riveros (democratacristiano, Presidente de la Comisión de Relaciones Exteriores de la Cámara de Diputados). Además, se entrevistó a Iván Witker, Alberto van Klaveren y Marcos Robledo. Es necesario tener presente que los recuerdos y testimonios de estos actores probablemente han sido mediados por el paso del tiempo, intencionalidades políticas y el consumo posterior de información de la cual entonces no se disponía. 
invasión a partir del análisis de las principales percepciones de Ricardo Lagos ${ }^{7}$.

Sin embargo, es preciso recalcar que la decisión en cuestión no fue una sola sino el resultado de un proceso secuencial de toma de decisiones relacionadas entre sí (Mintz y De Rouen Jr., 2010). En este artículo se analiza la primera (definición sustantiva) de las tres etapas que constituyeron el proceso de toma de decisiones ${ }^{8}$, que se extendió desde el ingreso de Chile al Consejo de Seguridad (1 de enero) hasta la exposición del Secretario de Estado Colin Powell ante el mismo organismo (5 de febrero). Fue durante este período que Lagos decidió no apoyar la invasión-35 días antes de ser comunicada a Estados Unidossobre la base de su impresión de que la guerra era inevitable y de que las

La principal limitación que enfrentó esta investigación fue la cercanía temporal del caso en estudio, que ha impedido acceder a los documentos de la época depositados en el Ministerio de Relaciones Exteriores. Además, no existen registros de las conversaciones y reuniones sostenidas por Lagos y su entorno que sean de acceso público. Estas restricciones de información han impedido realizar un análisis satisfactorio desde la perspectiva de una elección racional.

8 La segunda etapa (definición estratégica), en la cual se acomodó la decisión inicial y se coordinaron posiciones diplomáticas con México, se extendió entre el 5 de febrero y el 12 de marzo (última conversación Lagos-Bush). La tercera etapa (despliegue de la decisión), en la cual se dio a conocer públicamente la decisión de no apoyar la invasión y Chile presentó una propuesta de resolución propia, se extendió entre el 12 y el 19 de marzo, fecha en la que se inició la invasión. pruebas sobre la existencia de armas de destrucción masiva presentadas por Washington eran inconsistentes. Estas percepciones se confirmaron al comparar, por medio de razonamiento analógico, las exposiciones de Powell y del Embajador Adlai Stevenson durante la crisis de los misiles de 1962. La desconfianza de Powell y la ausencia de un remate en su presentación convencieron a Lagos de no apoyar la invasión.

El artículo se divide en dos grandes secciones. En la primera se examinan los factores que concentraron el poder decisorio en Lagos y, luego, las principales características de su visión del mundo. En la segunda se analizan las percepciones de Lagos en torno a la cuestión de Irak y la analogía PowellStevenson que las confirmó. Finalmente, presentamos nuestras conclusiones.

\section{RicARDO LAGOS COMO UNIDAD DECISORA}

La primera cuestión relevante que debe tenerse presente al analizar los procesos de toma de decisiones de política exterior es quién las toma, es decir, la unidad decisora. El tamaño y composición de esta puede influir de manera directa en la dinámica y resultados del proceso de toma de decisiones. Así, «no es esperable que el proceso de toma de decisiones llevado a cabo por una sola persona siga las mismas dinámicas que una decisión de grupo o una decisión de coalición. Si hay varios grupos involucrados, el proceso va a ser 
diferente de otros procesos» (Mintz y De Rouen Jr., 2010: 20).

Margareth Hermann (2001) define la unidad decisora como el grupo de actores ubicados en el ápice de la estructura de toma de decisiones que, si están de acuerdo, poseen la capacidad de comprometer los recursos del Estado en el sistema internacional y de impedir que otros actores internos reviertan abiertamente su posición. Existen tres tipos básicos de unidades decisoras: un líder predominante (un individuo capaz de suprimir los disensos y de ser necesario tomar decisiones por sí solo), un grupo único (personas pertenecientes a un mismo cuerpo que pueden definir un curso de acción en consulta entre sí) y una coalición de actores autónomos (personas que representan a distintas organizaciones que, en conjunto pero no por separado, poseen la capacidad de tomar decisiones) (Hermann, 2001).

Margareth y Charles Hermann (1989) identifican cinco condiciones que, de cumplirse, configuran un escenario en que hay grandes probabilidades de que la unidad decisora sea un líder predominante. Para efectos del análisis, agrupamos las condiciones en tres tipos de factores. Los factores político-institucionales se relacionan con la existencia de una persona capaz de comprometer los recursos del Estado en el sistema internacional pese a la oposición de otros actores internos. Los factores contextuales se refieren a si la situación de política exterior corresponde a una diplomacia de alto nivel o posee carácter crítico. El tercer grupo corresponde a los factores individuales y se relaciona con el hecho de que el individuo posea interés o experiencia en asuntos internacionales (Hermann y Hermann, 1989).

\subsection{Factores político-institucionales}

El fuerte presidencialismo del régimen político chileno, ampliamente descrito en la literatura pertinente, encuentra en la política exterior su expresión más nítida y radical, configurando lo que se ha denominado hiperpresidencialismo de la política exterior chilena (Fuentes Vera, inédito). El Presidente de la República posee la facultad exclusiva de conducir las relaciones exteriores del país sin contrapesos institucionales significativos ${ }^{9} \mathrm{o}$ actores que puedan o estén dispuestos a oponérsele. El carácter autoritativo de las decisiones de política exterior tomadas por el Presidente se ve reforzado por la actitud reactiva y abdicativa del Congreso Nacional, el alineamiento cuasi-automático de los partidos políticos respecto de las decisiones presidenciales y el desinterés y desinformación que expresan la ciudadanía y la opinión pública en torno a estos temas. La ausencia de un sistema de política exterior claramente definido (Van Klaveren, 2013) ha llevado a que las decisiones sean tomadas en un esquema de geometría variable que involucra «en grados diversos a los asesores del segundo piso, al Mi-

9 Ver en la Constitución Política de la República los Artículos $32^{\circ}$ (incisos $8^{\circ}$ y $15^{\circ}$ ) y $54^{\circ}\left(\right.$ inciso $1^{\circ}$ ). 
nisterio de Hacienda, al Canciller y a algunas instancias de la Cancillería, y a operadores de diversas agencias gubernamentales, quienes giran alrededor de una diplomacia presidencial poco institucionalizada, permeable a los lobbies y hasta cierto punto cerrada a la influencia del resto de la sociedad» (Fuentes Vera, inédito).

La persistencia de la idea de un consenso en torno a la política exterior contribuye a que las decisiones del Presidente, que posee la capacidad de comprometer los recursos del Estado prácticamente sin oposición interna, sean incontrarrestables.

\subsection{Factores contextuales}

Respecto del carácter crítico de la situación, el debate que precedió a la invasión de Irak ha sido una de las principales crisis internacionales del último tiempo, y ha marcado la agenda de la política mundial durante más de un decenio. La invasión, además, se enmarcó dentro de la guerra contra el terrorismo iniciada tras los atentados del 11 de septiembre de 2001, hecho que desde la perspectiva de Ricardo Lagos (2012) señaló un antes y un después en la política internacional. Para la diplomacia chilena, la cuestión de Irak adquiría ribetes particulares toda vez que, además de ser una situación novedosa, la posición que se adoptara podía tener un impacto negativo en las relaciones con su principal aliado estratégico-Estados Unidos-y el curso del tratado de libre comercio entre ambos.
El caso en estudio fue adquiriendo paulatinamente rasgos evidentes de una situación de diplomacia de alto nivel. Si bien en un principio los principales actores del debate fueron los representantes ante el Consejo de Seguridad, a medida que avanzaron las semanas, los ministros de relaciones exteriores y luego los jefes de Estado adquirieron mayor protagonismo mediante la participación en sesiones especiales del Consejo de Seguridad, en el primer caso, y conversaciones reservadas del más alto nivel así como encuentros públicos (como la Cumbre de las Azores), en el segundo.

\subsection{Factores individuales}

Ricardo Lagos es un político que podría calificarse de híbrido (Mella, 2010), a juzgar por una trayectoria que, combinando experiencia intelectual, diplomática y política, se aleja de los patrones tradicionales de los líderes de su generación ${ }^{10}$. Su fuerte carácter, la seguridad de sí mismo y su vehemencia lo han llevado a proyectar una imagen muchas veces de tozuda y autoritaria (Götz, 2010).

En materias de política exterior, Lagos se distinguió no solo por tomar las decisiones sino también por involucrarse de manera directa en su construcción, evidenciando su profundo interés y experiencia en política internacional.

10 Para un análisis de la carrera de Lagos y la conformación de su discurso, ver Mella (2010). Götz (2010) analiza la evolución de sus habilidades de liderazgo político. 
En efecto, las dos caracterizaciones existentes sobre su liderazgo de política exterior han puesto de relieve su personalismo en la toma de decisiones: Mariana Perry (2012) lo ha definido como un líder orientado hacia el objetivo y Shirley Götz (2010) como un lider directivo.

En línea con lo argumentado en este artículo, Götz (2010) afirma que el liderazgo directivo de Lagos se caracterizó por: a) la notoria personalización del diseño y conducción de la política exterior; b) el respeto de los límites que imponen la estructura de decisión formal y los medios interno y externo; c) las fuertes convicciones personales (visión de mundo) que moldean el proceso de toma de decisiones; y $d$ ) la construcción de un entorno decisional afín a dichas convicciones y estilo de trabajo $^{11}$.

\subsubsection{Lo internacional en la trayectoria política de Lagos}

Junto a Eduardo Frei Montalva (1964-1970), Ricardo Lagos ha sido uno de los presidentes chilenos más versados en política internacional (Fermandois, 2005; Götz, 2010). Desde temprana edad, su trayectoria intelectual fue permeada por los asuntos internacionales, frente a los cuales ha mostrado gran interés y reflexividad ${ }^{12}$.

11 El entorno decisional y el manejo presidencial de Lagos serán objeto de un futuro artículo.

12 Su primer recuerdo sobre lo público es la declaración de guerra que marcó el ingre-
Pese a tal precocidad, fue durante sus estudios de postgrado en Estados Unidos cuando comenzó a «descubrir el mundo y a mirarlo con otros ojos. [...] así fui aprendiendo a mirar Chile desde fuera de Chile» (Perry, 2012: 76).

Si bien a su regreso se incorporó a las labores académicas en la Universidad de Chile, Lagos comenzó a involucrarse cada vez más en la política activa. Después de varios años de haber renunciado al Partido Radical, afianzó sus vínculos con el Partido Socialista y Salvador Allende, quien tras su elección como Presidente lo nombró asesor económico y, posteriormente, representante ante diversas instancias multilaterales ${ }^{13}$ y embajador ante la Unión Soviética, cargo que no alcanzó a desempeñar debido al golpe de Estado de 1973. En el exilio, Lagos inició una carrera como funcionario internacional, vinculándose a organismos tales como el PNUD, PREALC y la UNESCO, entre otros. Además de permitirle generar lazos con importantes líderes euro-

so de Chile a la Segunda Guerra Mundial (1943) (Perry, 2012). Posteriormente, como Presidente de la Academia de Letras Castellanas del Instituto Nacional, pronunció un discurso sobre el cuerpo diplomático en Chile frente los principales embajadores en Santiago. En la universidad, pronunció un discurso en un homenaje al presidente guatemalteco Juan José Arévalo (Götz, 2010; Politzer, 1998).

13 XXVI Asamblea General de las Naciones Unidas (1971), Conferencia del Movimiento de los Países No Alineados (1971), III Conferencia sobre Desarrollo Económico y Comercio de las Naciones Unidas (1972) 
peos (Felipe González, Willy Brandt y Francois Miterrand, por ejempo), sus funciones internacionales lo insertaron en los principales debates de las ciencias sociales en América Latina, Asia, África y Europa (Götz, 2010). De acuerdo al propio Lagos, «ahí se formó una visión de mundo» (Perry, 2012: 70).

Después de algunos años de haber retornado a Chile, en 1983 Lagos fue elegido representante de la Convergencia Socialista en la Alianza Democrática que agrupaba a las fuerzas políticas opositoras al régimen autoritario que habían privilegiado la vía institucional para derrocarlo. El amplio reconocimiento a su liderazgo le permitió mantener y ampliar sus nexos internacionales. Un ejemplo de esto fue haber sido el representante chileno en la conmemoración del bicentenario de la toma de La Bastilla en 1989 (Politzer, 1998).

Una vez recuperada la democracia en 1990, la dilatada experiencia de Lagos generó especulaciones sobre su posible designación como Ministro de Relaciones Exteriores. Pese a que finalmente sus labores se concentraron en la política nacional, asumiendo como ministro de Educación (1990-1992) y de Obras Públicas (1994-1998), «en su ámbito más personal no había dejado de pensar, especialmente durante la década de los noventa, en este mundo de cambios que se venía encima tras el fin de la Guerra Fría y la caída del muro de Berlín» (Reyes Matta, 2009: 40). Así, en 1996 se inserta de manera activa en el debate sobre la renovación del pensamiento socialista sobre la globalización y las relaciones internacionales, pasando a ser parte del selecto Comité de 12 Miembros Distinguidos de la Internacional Socialista que derivaría prontamente en la Comisión Progreso Global, germen de la denominada Tercera Vía.

\subsubsection{Visión del mundo de Lagos}

La visión del mundo que moldeó la toma de decisiones de política exterior y el entorno más cercano a Lagos (Götz, 2010) puede sintetizarse en las cuatro dimensiones que se describen a continuación.

a) Moderación ideológica y Tercera Vía. Lagos formó su visión de la política a partir del rechazo a las concepciones ideológicas totalizantes y las visiones ortodoxas de la sociedad, sean de izquierdas o de derechas. Es probable que por ello, en distintos debates a lo largo de su carrera se haya ubicado en posiciones intermedias. Tales son los casos de su militancia en el Partido Radical (el tercio intermedio de los tres tercios de la política chilena hasta antes del golpe de Estado) durante su juventud y su liderazgo en el grupo de los suizos que buscaba reunificar el Partido Socialista en la década de 1980. Al consultársele si se define como marxista, Lagos ha respondido que "así no se define un hombre libre» (Politzer, 1998: 30).

Su participación a partir de la década de 1990 en el debate de la Tercera Vía fue otra expresión de su moderación ideológica. La Tercera Vía fue 
una corriente de pensamiento dentro de los líderes políticos e intelectuales socialdemócratas a nivel internacional que proponía la adopción de políticas económicas y sociales heterodoxas con el fin de superar la tradicional división entre izquierdas y derechas que, según Lagos (2012), es un anacronismo. Desde su punto de vista, esta corriente «apunta a mantener la estabilidad macroeconómica y promover el crecimiento sustentable basado en los mercados libres, implementando, al mismo tiempo, cambios sociales significativos, donde un Estado eficiente continúa jugando un papel clave» (Lagos, 2000: 22). Es posible embarcarse en la liberalización económica de la globalización y trabajar por la justicia social a nivel nacional (Lagos, 2012). Esta sería la base ideológica sobre la cual Lagos y su entorno más cercano construirían el programa de gobierno ( Crecer con igualdad») que lo llevaría a ser electo Presidente en el año 2000.

Si bien desde su perspectiva las políticas del Consenso de Washington no fueron erradas, resultaban insuficientes para hacer frente a los nuevos desafíos sociales de América Latina. En coherencia con los postulados de la Tercera Vía, Lagos se abocó durante los primeros años de su gobierno a promover un «nuevo consenso social internacional» que vinculara el avance material y el progreso social en la región (Lagos, 2000).

b) Sentido y conciencia histórica. Desde su infancia, como producto de la in- fluencia de su tío Ernesto, Lagos se interesó por el estudio de la historia, que se convertiría en su más grande afición. Según la periodista Patricia Politzer, él «se pasea por distintos siglos y latitudes con verdadera emoción. Aprovecha cualquier estímulo para profundizar algún tema o personaje» (Politzer 1998: 271). A partir de la historia, Lagos busca extraer «lecciones, experiencias y caminos para iniciar con un pie más firme el avance de nuestra sociedad en un mundo que será cada vez más global» (Lagos, 2010: 9). El análisis de los procesos políticos, económicos y sociales pretéritos constituye para Lagos una de las principales fuentes para interpretar el presente y abordar las tareas futuras. Debido a la gran importancia que para él tiene mirar hacia el futuro mientras que, al mismo tiempo, se aprende del pasado (Lagos, 2005), no es inusual que en distintos escritos, discursos y entrevistas, para respaldar sus argumentos se refiera a hechos y personajes de la historia de Chile y universal.

Su pasión por el pasado lo ha llevado a desarrollar una gran conciencia histórica y de la posición que él ocupa en los procesos políticos nacionales e internacionales. Lagos atribuía un sentido histórico particular a su asunción como Presidente, como producto de la convergencia de distintas circunstancias. En primer lugar, para él era de particular importancia el hecho de ser el primer presidente socialista desde la frustrada experiencia de Salvador Allende (19701973). Como ha apuntado Genaro 
Arriagada, Lagos estaba "obsesionado» con dejar «atrás el estigma que pesa sobre los socialistas de que pueden ganar elecciones pero no dar gobernabilidad» (Arriagada, 2012: 15).

En segundo lugar, su gobierno, además de ser el primero del siglo XXI, fue el penúltimo antes del bicentenario de la independencia de Chile, celebrado en 2010. Pensar el bicentenario y el tipo de sociedad a construir en el nuevo siglo, señalaba Lagos antes de asumir, sería la primera tarea de su gobierno (Politzer, 1998). A su juicio, el bicentenario era un momento propicio para evaluar el pasado, mirar el presente y proyectar el futuro. Más allá de los legados materiales, aspiraba a que su gestión sentara las bases sobre las cuales se construiría el tercer siglo de vida independiente del país (Lagos, 1999, 2010; Politzer, 1998 $)^{14}$.

En tercer lugar, desde su perspectiva, en distintos momentos históricos existen liderazgos que encarnan las fuerzas profundas y visiones de mundo (muchas veces en conflicto) que moldean el

14 Lagos ha afirmado que «Al asumir la Presidencia de Chile el 2000 me pareció que el país debía mirar y prepararse para el bicentenario del 2010. Se trataba no solo de establecer un horizonte hacia dónde desearíamos que estuviera Chile ese año, sino la ocasión de hacer de esta celebración un momento -más importante que las fiestas para solemnizar el acontecimiento- para mirar hacia atrás, para hacernos cargo de los errores, los avances, los orgullos que teníamos para mostrar, así como de las falencias que, en muchos campos, todavía presentaba la sociedad chilena» (Lagos, 2010; 7). devenir de una sociedad, sus avances y retrocesos (Lagos, 2010). Lagos sentía que la ciudadanía le asignaba un liderazgo de tipo histórico (Politzer, 1998) derivado de los roles que desempeñó a partir de los años ochenta, que le permitiría alcanzar sus ambiciosos fines. Sentía que había aportado al «tránsito de la dictadura a la democracia [... y] la consolidación de la Concertación como gobierno. Y hoy, creo que algo puedo aportar para moldear el Chile que vamos a construir a partir del próximo siglo» (Lagos en Politzer, 1998: 317),

En suma, Lagos no solo es un ávido observador de la historia sino también aspiraba a formar parte de ella. Pese a creer que en la mayoría de los casos la trascendencia de los líderes políticos no se compara con la de líderes espirituales y científicos (Politzer, 1998), subyacía en Lagos una preocupación respecto de la forma en que sería recordado su gobierno en los libros de historia. La proyección histórica de este fue el principal objetivo subyacente que orientó el trabajo del equipo estratégico del segundo piso de La Moneda (Campero, 2012).

c) Institucionalismo y sentido del deber. Según palabras del propio Lagos, durante su infancia y juventud fue formado con "una visión austera y responsable de la vida», en la cual el sentido del deber y el estudio formaban parte fundamental» (Politzer, 1998: 55). Estos valores, sumados a su formación inicial en derecho, influyeron de manera significativa en la centralidad 
que otorga al respeto a las instituciones y el cumplimiento del deber.

El respeto a las instituciones y la consolidación de la democracia fueron las ideas que orientaron la estrategia de gobierno diseñada por Lagos y su segundo piso. Si había algo por lo cual debía ser recordado su gobierno sería el fin de la transición democrática y la plena instauración del Estado de derecho (Campero, 2012). Ese fue el espíritu de reformas institucionales tales como la normalización de las relaciones cívicomilitares y la reforma constitucional de 2005, que pretendió otorgar una nueva Carta Fundamental para el Chile del siglo XXI. No obstante, la expresión más clara de este énfasis en las instituciones fue la expresión acuñada por Lagos, «en Chile, las instituciones funcionan», que parafrasearía hasta tiempos recientes.

El institucionalismo y el sentido del deber permearon también la visión de Lagos respecto del rol del Presidente en un régimen democrático, delimitando claramente las atribuciones y ámbitos de competencia de ese cargo y los que lo rodean. A su juicio, «los presidentes cumplen las funciones propias de los presidentes [...] Los presidentes hacen lo que deben hacer en función de su cargo. Los ministros hacen lo que deben hacer cuando son ministros. Y así lo hice cuando fui ministro» (Politzer, 1998: 150). En efecto, fue durante su gestión en el Ministerio de Educación cuando aprendió cómo se utiliza el aparato del Estado, el rol y los ámbitos de acción del Presidente y cómo éste de- lega en su equipo (Politzer, 1998). Para Lagos, el Presidente es quién conduce la nación y define los lineamientos fundamentales del gobierno a partir de una idea de país y una visión de futuro que interprete el sentir nacional. Esa idea de país se convierte «en un norte, en un horizonte nítido para tomar las decisiones adecuadas que permitan enfrentar dificultades, que ayude a avanzar con más claridad» (Lagos, 2005: s/p). En este sentido, su principal asesor en asuntos internacionales, el diplomático Fernando Reyes Matta, afirma:

«Lo dijo más de una vez en sus conversaciones con la prensa internacional: en el mundo de hoy se compite con todo lo que se es. Y la clave para ello estaba en tener un proyecto-país. Una visión del conjunto a donde se quiere llegar, un avanzar progresivamente mientras se busca hacer coincidir las piezas del puzzle» (Reyes Matta, 2009: 33).

Lagos creía tener su idea de país claramente definida y sobre esa base, durante la campaña presidencial justificaba su intención de gobernar el país (Politzer, 1998). Con el propósito de difundir su visión del país entre la ciudadanía, publicó, en marzo de 1999, un libro de campaña titulado Mi idea de país.

d) Globalización, politica exterior y desarrollo. La reflexión sobre la globalización ha permeado de manera significativa la visión de Lagos sobre la política y el desarrollo. Su participación activa en los debates de la Tercera Vía 
contribuyó a reforzar su convicción -desarrollada durante sus estudios de post-grado- según la cual, en último término, «el desarrollo de un país está inserto en el desarrollo del planeta del cual forma parte» (Lagos, 1993: 19). Lagos «creía conocer bien el mundo exterior y quería transformar ese conocimiento en políticas públicas y en una nueva orientación diplomática» (Rodríguez Elizondo, 2006: 263, citado en Götz, 2010).

En su opinión, «el crecimiento, el desarrollo social, la calidad de vida y la globalización son elementos complementarios y no contradictorios» (Lagos, 2001: s/p). Toda vez que el desarrollo de un país se vincula estrechamente con su capacidad para insertarse en la economía internacional, la política exterior constituye un instrumento fundamental que conecta los procesos políticos y económicos nacionales y globales.

De acuerdo con su formación de posgrado y la economización de la agenda internacional durante la última década del siglo XX, Lagos usualmente enfatizaba la dimensión económica de la globalización y las relaciones internacionales (Lagos 1993; 2000; 2001). ${ }^{15}$ En un mundo «donde las distancias se acortan y las fronteras se borran", Lagos afirma que «la interrelación entre

15 A partir de los atentados de septiembre de 2001, es posible observar en sus discursos un progresivo énfasis en la dimensión más política de la política internacional, el cual se consolida tras la participación de Chile en la cuestión de Irak. Ver Lagos (2005). las empresas, los países y las grandes regiones del mundo es progresiva y necesaria» (Lagos, 2001: s/p). Pese a reconocer que uno de los principales peligros asociados a la globalización es el crecimiento del movimiento de capitales, la globalización del mercado financiero seguirá acentuándose puesto que la globalización, en términos generales, es un proceso irreversible (Lagos, 2000).

Frente a este estado de cosas, la inserción eficiente y competitiva en la globalización es la única y más clara alternativa para el desarrollo de los países latinoamericanos (Lagos, 2000; 2001). La integración y el multipolarismo económico son dos de las principales tendencias asociadas a la globalización, y América Latina no puede quedarse al margen de estas. Esta inserción debe realizarse con base en el principio del regionalismo abierto, el cual «implica que los acuerdos comerciales entre agrupaciones de países no son incompatibles con aperturas comerciales más profundas, ya sean unilaterales, bilaterales o subregionales, y que incluso pueden encontrar un estímulo en estos últimos procesos» (Lagos, 2001: s/p). El horizonte de esta estrategia debe ser la «liberalización política y económica y la concreción de relaciones dinámicas con diversas regiones del orbe y esquemas de integración» (Lagos, 2001: s/p). 
3. Percepciones de Lagos en TORNO A LA INVASIÓN DE IRAK

Cuando ocurrieron los atentados del 11 de septiembre de 2001, Lagos se encontraba de gira por Europa. «Fue uno de esos días horribles en la historia: cuando el tiempo parece detenerse y el mundo cambia en 180 grados mientras todos observan incrédulos» (Lagos, 2012: 208). Sintiéndose parte de un proceso histórico fundamental, Lagos escribió-durante el vuelo que ese mismo día lo llevó desde Lisboa a Londres-algunas líneas sobre el cambio paradigmático al cual el mundo se veía enfrentado y lo que esto implicaría para los países libres del mundo, incluyendo Chile (Lagos, 2012). Al aterrizar, Lagos fue el primer líder mundial que expresó su apoyo a Estados Unidos ante la prensa.

Dos años más tarde, en septiembre de 2002, el gobierno chileno logró el apoyo del Grupo de Países Latinoamericanos y del Caribe (GRULAC) para ser parte del Consejo de Seguridad de las Naciones Unidas en el período 2003-2004. Con esto, Lagos buscaba, en primer lugar, equilibrar las dimensiones política y económico-comercial de la política exterior chilena, usualmente criticada por la gran publicidad de que era objeto esta última. En segundo lugar, Lagos creía que la participación en el Consejo era una oportunidad para «dar a conocer cuán lejos Chile había llegado, desde una dictadura sin control a un ejemplo de crecimiento económico y libertad» (Lagos, 2012:
207). En tercer lugar, subyacía en Lagos, en coherencia con su sentido del deber e institucionalismo, la creencia de que los países pequeños como Chile no pueden eludir el deber de contribuir a la preservación del orden multilateral. «La historia nos enseña que ante la ausencia de reglas compartidas, los intereses de los más poderosos prevalecen. Esa es la razón que subyace a por qué [...] Chile entendió que su primer deber era fortalecer las instituciones multilaterales» (Lagos, 2005: 18). Finalmente, y vinculado con lo anterior, el ingreso al Consejo resultaba funcional a su pretensión de erigirse como un líder regional que ejerciera como bastión de la Tercera Vía en América Latina una vez que Fernando Henrique Cardoso dejara el poder ${ }^{16}$. La promoción del nuevo consenso social internacional era parte de dicha estrategia (Bywaters, 2013).

\subsection{La definición del problema según Lagos}

Para Lagos, el núcleo del debate en torno a la invasión de Irak era si Estados Unidos necesitaba o no una nueva resolución que autorizara de manera explícita el uso de la fuerza contra el régimen de Hussein, además de la Resolución 1441 del 8 de noviembre de 2002. «Problema: hay una resolución del mes de noviembre. Típica resolución negociada por diplomáticos donde cada uno interpreta lo que quiere interpretar [...] Esa fue la forma de lograr la reso-

16 Entrevista con asesor de Lagos. 
lución, claro. Cada uno iba a plantear lo que quería» (Lagos, 2013: s/p). A su juicio, la resolución era "convenientemente maleable $[. .$.$] todos podían re-$ fugiarse en ella porque significaba todo y nada» (Lagos, 2012: 208). En torno a este eje, identificaba dos posturas en contradicción:

«Para los estadounidenses, era suficiente prometer consecuencias por la falta de cumplimiento de Saddam, aunque sin plazos firmes. ¿Qué otro lenguaje podrían necesitar para ir a la guerra? En la diplomacia internacional, todos saben lo que significa consecuencias. Para los franceses, meramente significaba que Saddam tenía que cumplir, pero como no se establecían ni consecuencias firmes o plazos, ir a la guerra claramente requeriría otra resolución» (Lagos, 2012: 208).

En este marco, Lagos (2012) creía que Chile se encontraba en una posición desfavorable, propia de los miembros no permanentes del Consejo y que los permanentes suelen no entender. En una conversación telefónica con el Presidente Jacques Chirac (24 de febrero), Lagos retrató esta posición como sigue:

«Señor Presidente [...] ¿Quiere saber cuál es la diferencia entre usted y yo? [...] La diferencia es que usted tiene un voto y un veto [...] Si se abstiene, Bush lo llamará y le agradecerá al día siguiente. Como Presidente de Chile, solo tengo un voto. Si me abstengo, Bush no obtendrá los nueve votos que necesita» (Lagos, 2012: 220) ${ }^{17}$.

17 Este argumento está presente también en Lagos (2003).
Esta posición reducía el margen de acción de los miembros no permanentes, particularmente aquellos que son pequeños, dejándoles básicamente dos opciones: la más común, que es votar de acuerdo al parecer de alguna gran potencia, o poseer una posición autónoma en la política internacional y defenderla (Lagos, 2005).

Si bien ha afirmado que nunca se sintió presionado por Estados Unidos para votar de una forma u otra (Lagos, 2012), por distintos medios Washington hizo saber al gobierno chileno que una posición contraria a sus intereses pondría en riesgo el acuerdo comercial. Tal fue la percepción del director general de Política Exterior Carlos Portales en reuniones del Comité de la Democracia (Portales, 2012) y del ministro Heraldo Muñoz (2004) en sus conversaciones con Condoleeza Rice. Frente a esta posibilidad, Lagos mantuvo una actitud confiada puesto que le parecían dos asuntos inconexos. Pocos meses después, afirmó: «si tenemos un acuerdo de libre comercio es porque beneficia a ambos países, y la cuestión de cómo votaremos en el Consejo de Seguridad debería ser un asunto sin relación» (Lagos, 2003: 124). Además, pareciera ser que la no suscripción del tratado era un costo que estaba dispuesto a asumir, aunque veía tal escenario como improbable.

«Yo no estaba asustado en lo absoluto por el acuerdo de libre comercio. [...] Si Estados Unidos quería que no se firmara, pues no se firmaba. Pero mi pregunta era: ¿y en qué queda la 
política externa de Estados Unidos, que lo único que tiene para mostrar es que ha firmado un acuerdo con Chile?» (Lagos, 2013: s/p).

Lagos y su entorno más cercano eran conscientes, además, de la limitante de tiempo que representaba el inicio de la primavera en Irak: el árido, ventoso y caluroso clima del desierto dificultaría la operatividad del ejército estadounidense, por lo cual el Pentágono no esperaría más allá de la tercera semana de marzo para iniciar la invasión (Muñoz, 2005; Valdés, 2013) ${ }^{18}$.

\subsection{Principales percepciones de Lagos}

\subsubsection{Inevitabilidad de la guerra}

Esta percepción se formó tempranamente, desde el momento en que Chile ingresó al Consejo de Seguridad.

18 Algunas interpretaciones han apuntado a que esta variable habría tenido un peso significativo en la definición de la estrategia chilena, la cual se habría orientado a dilatar la discusión hasta entrado marzo para evitar la votación de una segunda resolución enmendada propuesta por Estados Unidos, situación que habría puesto a Chile en abierta contradicción con Washington. Si bien sobre la base de la información recopilada en esta investigación no es posible afirmar o refutar dicha hipótesis, frente a esta cuestión Lagos ha afirmado: «No, nada que ver. Eso a mí no me preocupó nunca. Nunca me preocupó cómo íbamos a votar, primero, porque sabía cómo íbamos a votar. Eso no me desveló ni un minuto; eso se lo garantizo» (Lagos, 2013: s/p).
"Cuando [Juan Gabriel] Valdés tomó asiento en el Consejo de Seguridad por primera vez en enero de 2003, ya sentíamos la presión. Estados Unidos se estaba preparando para la guerra [...] Era como si la suerte de las cuestiones ante nosotros ya estaba decidida. Estábamos discutiendo una nueva resolución sobre las supuestas armas de destrucción masiva de Irak. Pero Bush ya había decidido ir a la guerra. Estábamos luchando para detener un conflicto que Bush creía que era inevitable. Y, al fin y al cabo, quizá él tenía razón: la guerra no podía ser evitada porque Bush era quien manejaba los hilos. La convicción estadounidense era una profecía auto-cumplida respecto a la cual podíamos hacer poco» (Lagos, 2012: 212).

Diversas fuentes contribuyeron a crear esta percepción. La primera de ellas fue la información transmitida por el Embajador Juan Gabriel Valdés entre septiembre y diciembre de 2002. Durante ese período, Valdés (2012) se formó la impresión de que Estados Unidos invadiría Irak con o sin el consentimiento de las Naciones Unidas. Fundamental en el desarrollo de esta fue la invitación ${ }^{19}$ que recibió para ser puesto al corriente de la posición estadounidense en torno a Irak en el Foggy Bottom ${ }^{20}$. La incoherencia de los

19 No se ha podido determinar la fecha exacta de este evento.

20 Foggy Bottom es un barrio de la ciudad de Washington D.C. donde se ubican diversas embajadas, organismos internacionales y el Departamento de Estado. 
argumentos presentados eran, desde su punto de vista, expresión del voluntarismo estadounidense que hacía que la guerra fuera inevitable (Valdés, 2012). Valdés transmitió estas percepciones a Lagos durante el último trimestre de 2002, por lo cual puede colegirse que este ya poseía un juicio medianamente formado al momento de ingresar al Consejo de Seguridad ${ }^{21}$.

La segunda fuente fue la interpretación realizada por Heraldo Muñoz a partir de la conversación sostenida el día 21 de enero con su amiga y Consejera de Seguridad Nacional, Condoleeza Rice. En una llamada telefónica, Rice señaló a Muñoz:

«Heraldo, el tiempo se está acabando. Lo máximo que tenemos son dos o tres semanas antes de que iniciemos la guerra contra Saddam [...] espero que puedas ayudarnos y discutir esto con el Presidente Lagos. Pero iremos adelante con o, si es necesario, sin el Consejo de Seguridad de la ONU» (Muñoz, 2005: 19-20).

Muñoz interpretó esas palabras como la voluntad clara de embarcarse en la invasión. "Acabada la conversación, esta última frase me quedó resonando. Si fuese necesario, Estados Unidos se lanzaría solo a invadir Irak. La guerra era inevitable pensé» ( $\mathrm{Mu}-$ ñoz, 2005: 20). Tras la conversación,

$21 \quad$ Las mismas pruebas presentadas a Valdés en el Foggy Bottom fueron las que los agentes de inteligencia mostraron a Lagos el 24 de febrero en Santiago (Lagos, 2012; Valdés, 2012). Otro registro del evento en Muñoz (2005).
Muñoz comentó sus percepciones a Lagos, quien le pidió intercambiar comentarios luego de la conversación telefónica con Bush que se concretaría al día siguiente.

De acuerdo con el testimonio de Lagos (2012), en dicha conversación-la tercera fuente-Bush fue directo al grano: creía que la Resolución 1441 era suficiente para iniciar la invasión. Irak fue el único tema del intercambio (Muñoz, 2005). Si bien Lagos compartía las preocupaciones sobre la seguridad y el terrorismo internacional que motivaban a Bush, "no podía ver la conexión con Irak» (Lagos, 2012: 14). Tras la conversación, Muñoz afirma que «Lagos se mostró pesimista sobre la situación internacional. Con una expresión de resignación me comentó: obviamente, la situación se ve muy mala. Inmediatamente después, Lagos, con voz serena, agregó: creo que habrá guerra» (Muñoz 2005: 23).

\subsubsection{Inconsistencia de las pruebas estadounidenses}

Desde un principio, Lagos se mostró dudoso de la existencia de armas de destrucción masiva en Irak. Esta impresión se formó a partir de tres fuentes. La primera fue la inconsistencia de las pruebas mostradas a Valdés en el Foggy Bottom. En el encuentro, exhibieron un mapa que indicaba la supuesta localización de las armas en bases subterráneas en pleno desierto iraquí. Las misiones de inspección de la UNMOVIC, según los personeros estadounidenses, no las 
habían hallado debido a la capacidad del ejército iraquí para trasladarlas de un lugar a otro antes de las inspecciones. Ironizando, Valdés preguntó cómo era posible tal hecho considerando las altas temperaturas de esa zona y el mal estado y la desmoralización de las tropas iraquíes (Valdés, 2012). Lagos (2012) insinúa que estaba al tanto de los argumentos presentados a Valdés antes de la exposición de Powell del 5 de febrero y que estos habrían sido prácticamente idénticos.

La segunda fuente fueron las contradicciones entre las afirmaciones estadounidenses y los informes de los inspectores de la UNMOVIC, liderados por el diplomático sueco Hans Blix. El equipo de Blix presentó un informe ante el Consejo de Seguridad el día 27 de enero en el cual afirmaba que no se había encontrado evidencia que permitiera afirmar la existencia del armamento. Frente a esto, el gobierno chileno apostó por fortalecer y otorgar más tiempo a las inspecciones. Dos días después, Colin Powell solicitó una sesión especial del Consejo con la presencia de los ministros de relaciones exteriores para mostrar pruebas irrefutables sobre la existencia de armas de destrucción masiva en Irak. Entre enero y marzo, Lagos habló en al menos dos oportunidades con Blix (Lagos, 2012, 2013; Valdés, 2012), recibiendo información de primera fuente. Valdés, además, conversó en diversas ocasiones con Blix, con quien tenía especial cercanía debido a una antigua amistad con la hija del sueco (Valdés, 2012).
La tercera fuente fue la respuesta evasiva que Bush dio a Lagos cuando este le preguntó respecto de las armas de destrucción masiva en una conversación telefónica del 22 de enero. Lagos recuerda la conversación como sigue:

«Señor Presidente, ¿̇usted cree que Saddam Hussein estuvo involucrado en el 11 de septiembre?

No, respondió Bush. Pero su régimen paria... todo estuvo imbricado para apoyar el terrorismo.

Intenté otra forma de preguntar. ¿Está seguro de que hay armas de destrucción masiva? ¿Qué tan buena es su inteligencia?» (Lagos, 2012: 214).

Bush no respondió: solo se ciñó a su libreto (Lagos, 2012), casi idéntico al que, el día anterior, Condoleeza Rice había ocupado con Muñoz (2004). Según Lagos, tras la conversación «comenzó una acumulación de tensiones que terminó en la presentación de Powell, que se suponía que probaría a dudosos como yo que en realidad sí había armas de destrucción masiva en Irak» (Lagos, 2012: 215).

\subsection{Analogía Powell-Stevenson}

Los seres humanos recurrimos a diario al uso del razonamiento analógico para interpretar la información que recibimos de nuestro entorno y tomar decisiones a partir de ella, y los decisores en materia de política exterior no escapan a este hecho (Houghton, 1996). No obstante, mientras que en los estudios de psicología cognitiva 
hay acuerdo en que el razonamiento no solo se basa en modelos analógicos, los escritos sobre política exterior han tendido a partir de la base de que los encargados de tomar decisiones razonan primariamente por analogía, sin poner atención al uso de razonamiento abstracto (Breuning, 2003). Los decisores recurren a las analogías cuando «describen un evento como similar a un evento previo, a menudo atando a esa analogía un curso de acción específico que debería ser seguido para lograr un resultado esperado, o evitado para prevenir un resultado indeseado» (Breuning, 2003: 230).

Respecto al modo en que se comparan analógicamente dos eventos, David P. Houghton afirma que el decisor, esperando encontrar similitudes en algunos o todos sus atributos, «identifica una situación pasada (o base analógica) que parece particularmente útil para entender la naturaleza de una situación presente (o blanco analógico); la base luego es trazada ${ }^{22}$ en el blanco» (Houghton, 1996: 541).

Bush llamó a Lagos inmediatamente después de la presentación de Powell ante el Consejo de Seguridad. Ante la pregunta de qué le había parecido, el Presidente chileno respondió: «hubo momentos interesantes» (Lagos, 2012: 206). La presentación no lo había convencido pero creía que no era el momento para hacérselo saber a su par estadounidense. Según Lagos, «lo que me daba vueltas en la cabeza era

22 En el original, mapped. el escalofriante contraste entre las observaciones de Powell y las de [Adlai] Stevenson» (Lagos, 2012: 206) ${ }^{23}$ durante la crisis de los misiles.

Como se ha señalado, Lagos confirmó sus percepciones de que la guerra era inevitable y que no existían pruebas concluyentes sobre la existencia de armas de destrucción masiva en Irak al comparar analógicamente las exposiciones de Powell (blanco analógico) y Stevenson del 25 de febrero de 1962 (base analógica) ante el Consejo. Ambas presentaciones tenían por objeto dar a conocer pruebas irrefutables de la existencia de armas de destrucción masiva en Irak y Cuba, respectivamente. Mientras la exposición de Stevenson fue el punto de inflexión que dio la razón a Estados Unidos, la de Powell no logró el mismo resultado.

El uso de esta base analógica no fue casual: Lagos estaba al tanto de que «en los corredores de Naciones Unidas, todo el mundo terminó haciéndose la idea de que esto iba a ser Stevenson II» (Lagos. 2013: s/p). Si bien la presentación de Stevenson marcó a muchos interesados en las relaciones internacionales de su generación, probablemente tuvo un impacto mayor en Lagos, quien se encontraba por entonces realizando sus estudios de postgrado en Estados Unidos $^{24}$. En 1962, además, Chile

$23 \quad$ Subrayados en las citas subsecuentes por el autor.

24 Stevenson no era un personaje desconocido en la política estadounidense de aquella época: además de haber sido embajador y Gobernador de Illinois (1949- 
también era miembro del Consejo de Seguridad.

Como se ha argumentado, antes del inicio de la exposición, Lagos ya se había formado la impresión de que la guerra era inevitable. «Por entonces, era más que claro lo que Bush buscaba con el discurso de Powell. Estados Unidos iría a la guerra y si el mundo se convencía de unirse, sería simplemente un bono» (Lagos, 2012: 206).

a) Base analógica: Stevenson 1962. La imagen que Lagos tiene de Adlai Stevenson ha sido mediada por información adquirida posteriormente. Lagos lo recuerda así:

«Hay en algún libro que leí de Kennedy en que salía que Kennedy (sic), cuando vio esta intervención [de Stevenson en el Consejo de Seguridad], dijo: Bah, no sabía que el sabio tenía también mucha pasión. El cabeza de huevo le decía a Stevenson. Claro, era un intelectual, un hombre sofisticado en cuanto a su inteligencia, razón por la cual nunca pudo llegar a ser Presidente de Estados Unidos» (Lagos, 2013: s/p).

Respecto de la exposición ante el Consejo de Seguridad, Lagos afirma que «lo de Stevenson fue muy convincente» (Lagos, 2013: s/p). Lagos recuerda la presentación como sigue:

«Cuando él [Stevenson] te muestra fotos de un barco que va navegando y que su cubierta están los misiles, y le dice al embajador soviético: jéstas son las fotos! ¡No estoy mintiendo!

1953), fue candidato a la Presidencia de Estados Unidos en 1952 y 1956.
¡Responda!. Y él la única respuesta que le da es Perdón señor, no estoy en un tribunal de la Corte de Justicia de los Estados Unidos. ¿Quién se cree usted para preguntarme esta impertinencia?

Entonces el otro se enoja más y le contesta: ¡Por favor, señor, responda! ¿Quedaré esperando su respuesta hasta que el diablo se derrita en el infierno!» (Lagos, 2013: s/p).

\section{b) Blanco analógico: Powell 2003.} Consultado respecto de la credibilidad de Powell, Lagos afirma: "Yo creo que él la tenía. Lo que él no tuvo fue esa presentación que hizo. Era un hombre que tenía una imagen de honestidad intelectual. O sea, que era creíble» (Lagos, 2013: s/p).

La exposición de Powell no solo no llamó su atención (Lagos, 2013) sino que no lo dejó para nada convencido (Lagos, 2012). Lagos recuerda que la voz de Powell tenía un tono de seriedad y que su postura era solemne, aunque no lo veía del todo confiado (Lagos, 2012).

«Superficialmente, el discurso de Powell, sus movimientos, su tono grave y su argumento evocaban un momento icónico en la historia de Naciones Unidas: Adlai Stevenson [...] dejando en vergüenza a la Unión Soviética con evidencia clara de su traición al enviar armas nucleares a Cuba. En contraste, la evidencia de Powell no pudo haber sido menos convincente. Lo que verán es una recopilación de hechos e inquietantes patrones de conducta, Powell advirtió al Consejo. Pero nunca vimos ese remate» (Lagos, 2012: 206). 
Cristóbal Bywaters • El «NO» de Ricardo Lagos a la invasión a Irak en 2003...

c) Trazado analógico. Lagos percibió un gran contraste entre las presentaciones de Powell y Stevenson. Al analogarlas, Lagos identificó similitudes y diferencias entre ambas. El Cuadro 1 compara los atributos observados a partir de tres variables: imagen del expositor, forma de la presentación y contenido de la presentación.

En lo que se refiere a los expositores, Lagos cree que Stevenson era una persona destacada por una inteligencia sofisticada; un intelectual. Por otra parte, identifica a Powell como un hombre dotado de honestidad intelectual y, por lo tanto, creíble. Es posible inferir que también asigna este último atributo a Stevenson, toda vez que establece una relación entre intelecto y credibilidad. En suma, las imágenes que Lagos poseía de ambos emisores eran similares.

\section{Cuadro 1}

Cuadro comparativo de atributos observados en las presentaciones de Stevenson y Powell

\begin{tabular}{|c|c|c|}
\hline $\begin{array}{c}\text { Atributos } \\
\text { observados }\end{array}$ & $\begin{array}{c}\text { Base analógica } \\
\text { (Stevenson 1962) }\end{array}$ & $\begin{array}{c}\text { Blanco analógico } \\
\text { (Powell 2003) }\end{array}$ \\
\hline $\begin{array}{l}\text { Imagen del } \\
\text { expositor }\end{array}$ & $\begin{array}{c}\text { - Intelectual } \\
\text { - Inteligencia sofisticada. }\end{array}$ & $\begin{array}{c}\text { - Credibilidad } \\
\text { - Honestidad intelectual. }\end{array}$ \\
\hline $\begin{array}{l}\text { Forma de la } \\
\text { presentación }\end{array}$ & $\begin{array}{l}\text { - Voz grave, pasión, convicción. } \\
\text { - «Muy convincente». }\end{array}$ & $\begin{array}{l}\text { - Voz grave; tono de seriedad; } \\
\text { postura solemne. } \\
\text { - Movimientos similares a la de } \\
\text { Stevenson. } \\
\text { - «No del todo confiado». }\end{array}$ \\
\hline $\begin{array}{l}\text { Contenido de la } \\
\text { presentación }\end{array}$ & - «Convincente» & $\begin{array}{l}\text { - «No pudo haber sido menos } \\
\text { convincente». } \\
\text { - Argumentos similares a los de } \\
\text { Stevenson. } \\
\text { - «No hubo remate». }\end{array}$ \\
\hline
\end{tabular}

Fuente: elaboración propia a partir de Lagos (2012, 2013).

Sobre la forma de las exposiciones, Lagos observó en Stevenson atributos asociados a la convicción y la pasión que lo llevaron a creer que «fue muy convincente» (Lagos, 2013: s/p). En lo que respecta a la presentación de Powell, pese a identificar movimientos y tono de voz similares a Stevenson y observar una postura solemne y un tono de seriedad, Lagos percibió que el expositor no estaba del todo confiado.

Al referirse al contenido de las presentaciones, Lagos parece identificar en las pruebas presentadas por Stevenson (las fotografías de barcos con misiles en sus cubiertas en dirección a Cuba) atributos vinculados a la irrefutabilidad que lo llevaron a formarse el juicio de que eran convincentes. Pese a sentir que los argumentos de Powell evocaban los de Stevenson, Lagos afirma que la presentación de 2003 «no pudo haber sido menos convincente» (Lagos, 2012: 206). Las expectativas creadas por el gobierno estadounidense y el mismo Bush, quien pidió al Presidente chileno 
que viera la presentación, llevaron a que Lagos no percibiera algún "remate» que estableciera la irrefutabilidad de las pruebas. Como el mismo Lagos (2012) insinúa, las pruebas presentadas por Powell no fueron muy distintas de las presentadas a Valdés meses antes en el Foggy Bottom.

\section{Conclusión}

La decisión chilena de no apoyar la invasión estadounidense a Irak fue tomada por el Presidente Ricardo Lagos 35 días antes de su inicio. Diversos factores contribuyeron a que el poder de toma de decisiones de política exterior se concentrara en la figura del Presidente, tales como el hiperpresidencialismo de la política exterior chilena (factores político-institucionales), el carácter crítico y de diplomacia de alto nivel del proceso previo a la invasión (factores coyunturales) y el amplio interés y experiencia de Lagos en asuntos de política internacional (factores individuales). Respecto a este último punto, el amplio bagaje de Lagos en asuntos internacionales, su concepción sobre el rol del Presidente de la República y el hecho de tener una percepción consolidada del mundo fueron factores que reforzaron el carácter autoritativo de la decisión presidencial y la centralidad de Lagos en el proceso de toma de decisiones.

Lagos percibió tempranamente que la guerra era inevitable y que en Irak no existían pruebas claras sobre la existencia de armas de destrucción masiva. Estas percepciones se formaron a partir de diversas fuentes, entre las cuales cabe destacar la información que le proporcionó Juan Gabriel Valdés a partir del último trimestre de 2002 y la información transmitida por Condoleeza Rice al ministro Heraldo Muñoz. Lagos confirmó sus percepciones al comparar, por medio de razonamiento analógico, las exposiciones del Secretario de Estado Colin Powell, que pretendían dar a conocer pruebas irrefutables sobre la supuesta amenaza iraquí, con la presentación del Embajador Adlai Stevenson ante el Consejo de Seguridad de las Naciones Unidas con ocasión de la crisis de los misiles en 1962. Si bien Lagos tenía una buena imagen de ambos diplomáticos y la exposición de Powell le recordó la de Stevenson, la inseguridad y la ausencia de un remate en la exposición del primero terminaron por convencerlo de la inconveniencia e inviabilidad de apoyar la invasión.

Persisten, no obstante, algunas interrogantes sobre el caso en estudio que pueden abrir nuevas líneas de investigación al respecto. Resulta de particular interés indagar en mayor profundidad en las relaciones de la misión chilena en las Naciones Unidas con sus símiles del Consejo de Seguridad y las de otros países. En este sentido, la coordinación diplomática chileno-mexicana resulta fundamental para una comprensión más cabal del caso, particularmente desde la perspectiva de México. Por cierto, la progresiva apertura y desclasificación de archivos reservados espera- 
ble para los próximos años constituirán una oportunidad para profundizar en la comprensión del caso en estudio.

Esta investigación aspira a contribuir a una línea de investigación emergente que analiza el impacto de las fuentes internas en la política exterior de Chile. Si bien desde hace décadas es un hecho aceptado que la política nacional influye en las orientaciones y decisiones de un Estado en el ámbito externo, el nivel de penetración de los enfoques que analizan este tipo de variables ha sido escaso en el análisis de nuestra política exterior. La academia nacional ha tendido a presumir que, tal como argumentan quienes la diseñan, la política exterior es una política de Estado en torno a la cual no existirían disensos ni conflictos, cuestión bastante alejada de la realidad. No es deseable que los expertos en relaciones internacionales acepten estas premisas de manera acrítica y acepten el discurso político como si fuera una verdad científica.

\section{Bibliografía}

Arriagada, Genaro (2012), «Introducción: ¡Bienvenidos a la presidenciología!», en Fernández, María de los Ángeles y Eugenio Rivera (eds.), La trastienda del gobierno. El eslabón perdido en la modernización del Estado chileno. Santiago: Catalonia.

Breuning, Marijke (2003), «The role of analogies and abstract reasoning in decisionmaking: evidence from the debate over Truman's proposal for development assistance», International Studies Quarterly, $\mathrm{n}^{\circ} 47$, pp. 229-245.
Bywaters, Cristóbal (2013), «La posición de Chile en el debate en torno a la invasión a Irak en el Consejo de Seguridad en 2003. Principios, intereses y objetivos de la política exterior», en: Correa, Loreto (ed.), Ensayos de política exterior contemporánea, Santiago : ADICA.

Campero, Guillermo (2012). Entrevista con el autor realizada el 16 de agosto de 2012.

Di Lecco, Enrique (2005). Estudio de la política comercial de Chile durante los gobiernos de la Concertación, desde la perspectiva de las políticas públicas. (Tesis de Magíster en Ciencia Política) Universidad de Chile.

Errázuriz, Hernán F. (2003), «La diplomacia chilena en la segunda guerra de Irak», Estudios Internacionales, vol. XXXVI, n¹42, pp. 113-120.

Fermandois, Joaquín (2005), Mundo y fin de mundo: Chile en la política mundial 1900-2004, Santiago : Universidad Católica de Chile.

Fuentes Vargas, Cristián (inédito), La política exterior de Chile como política pública. Facilitado por el autor.

Götz, Shirley (2010). Liderazgo y política exterior chilena: Una interpretación de los cambios de la política regional en los gobiernos de Lagos y Bachelet. (Tesis de Magíster en Ciencia Política) Pontificia Universidad Católica de Chile.

Hermann, Margareth (2001), «How decision units shape foreign policy: A theoretical framework», International Studies Review, vol. 3, no. 2, Summer, pp. 47-81.

Hermann, Margareth (2002), Assessing leadership style: A trait Analysis. Recuperado el 13 de junio de 2013 de http://socialscience.net/docs/lta.pdf

Hermann, Margareth y Charles Hermann (1989), «Who makes foreign policy decisions and how: An empirical enquiry», International Studies Quarterly, vol. 3, no. 4, pp. 361-387.

Houghton, David P. (1996), «The role of analogical reasoning in novel foreign-policy situations", British Journal of Political Science, vol. 26, no. 4, pp. 523-552. 
Lagos, Ricardo (1993), «Visión del mundo y política internacional», extractos del discurso pronunciado en el CARI el 25 de enero de 1993. Buenos Aires : Argentina.

Lagos, Ricardo (1999), Mi idea de país, Santiago : Prosa.

Lagos, Ricardo (2000), «Hacia un nuevo consenso social internacional», en Lagos, Ricardo y Heraldo Muñoz (eds.), Globalización XXI: América Latina y los desafíos del nuevo milenio, Santiago, Aguilar.

Lagos, Ricardo (2001), "Chile en un mundo en cambio", Foreign Affairs en Español, $n^{\circ} 1$, Primavera, pp. 67-81.

Lagos, Ricardo (2003), "A view from the South», discurso pronunciado en la Mesa Redonda de la Conferencia de la Gobernanza Global el 12 de julio de 2003 en Londres, Reino Unido. En Lagos, Ricardo (2005), The 21st Century: A view from the South, London : First.

Lagos, Ricardo (2005), The 21st Century: A view from the South, London : First.

Lagos, Ricardo (2010), Cien años de luces y sombras, volumen I, Santiago : Taurus.

Lagos, Ricardo (2012), The southern tiger: Chile's fight for a democratic and prosperous future, Nueva York : Palgrave Macmillan.

Lagos, Ricardo (2013). Entrevista con el autor realizada el 12 de junio de 2013.

Mella, Marcelo (2010). El eclecticismo no resuelto del liderazgo de Ricardo Lagos, DAAPGE, $n^{\circ} 14$, pp. 1-30.

Mintz, Alex y Karl De Rouen Jr., (2010), Understanding foreign policy decision making, Nueva York : Cambridge University Press.

Muñoz, Heraldo (1986), Las relaciones exteriores del gobierno militar chileno, San- tiago : PROSPEL-CERC - Las ediciones del ornitorrinco.

Muñoz, Heraldo (2005), Una guerra solitaria. La historia secreta de EE.UU. en Irak, la polémica en la ONU y el papel de Chile, Santiago : Debate.

Núñez, Ricardo (2012). Entrevista con el autor realizada el 6 de julio de 2012.

Perry, Mariana (2011), La personalidad de Ricardo Lagos como factor determinante en la política exterior de Chile 2000-2006. (Tesis de Magíster en Estudios Internacionales) Universidad de Chile.

Politzer, Patricia (1998), El libro de Lagos, Santiago : Ediciones Grupo Zeta.

Porras, José I. (2003), La estrategia chilena de acuerdos comerciales: Un análisis político, Serie Comercio Internacional, $n^{\circ} 36$, Santiago : CEPAL.

Reyes Matta, Fernando (2013). Entrevista con el autor realizada el 11 de junio de 2013.

Rodríguez Elizondo, José (2006), Las crisis vecinales del gobierno de Lagos, Santiago : Debate.

Ross, César (2005), Chile y Japón 1973-1989: De la incertidumbre a la alianza estratégica, Santiago : LOM.

Valdés, Juan Gabriel (2008), La marcha de la locura. Recuperado el 6 de junio de 2013 de http://blogs.publico.es/dominiopublico/ category/juan-gabriel-valdes/.

Valdés, Juan Gabriel (2012). Entrevista con el autor realizada el 1 de mayo de 2012 .

Van Klaveren, Alberto (2013). Entrevista con el autor realizada el 3 de mayo de 2013.

Walker, Ignacio (2006), «La política exterior chilena", Estudios Internacionales, vol. XXXIX, n ${ }^{\circ} 155$, pp. 9-35. 